\title{
Shape Matters: Creating Amorphous Semiconductors
}

\section{Key words}

semiconductors

amorphous materials

chromophores

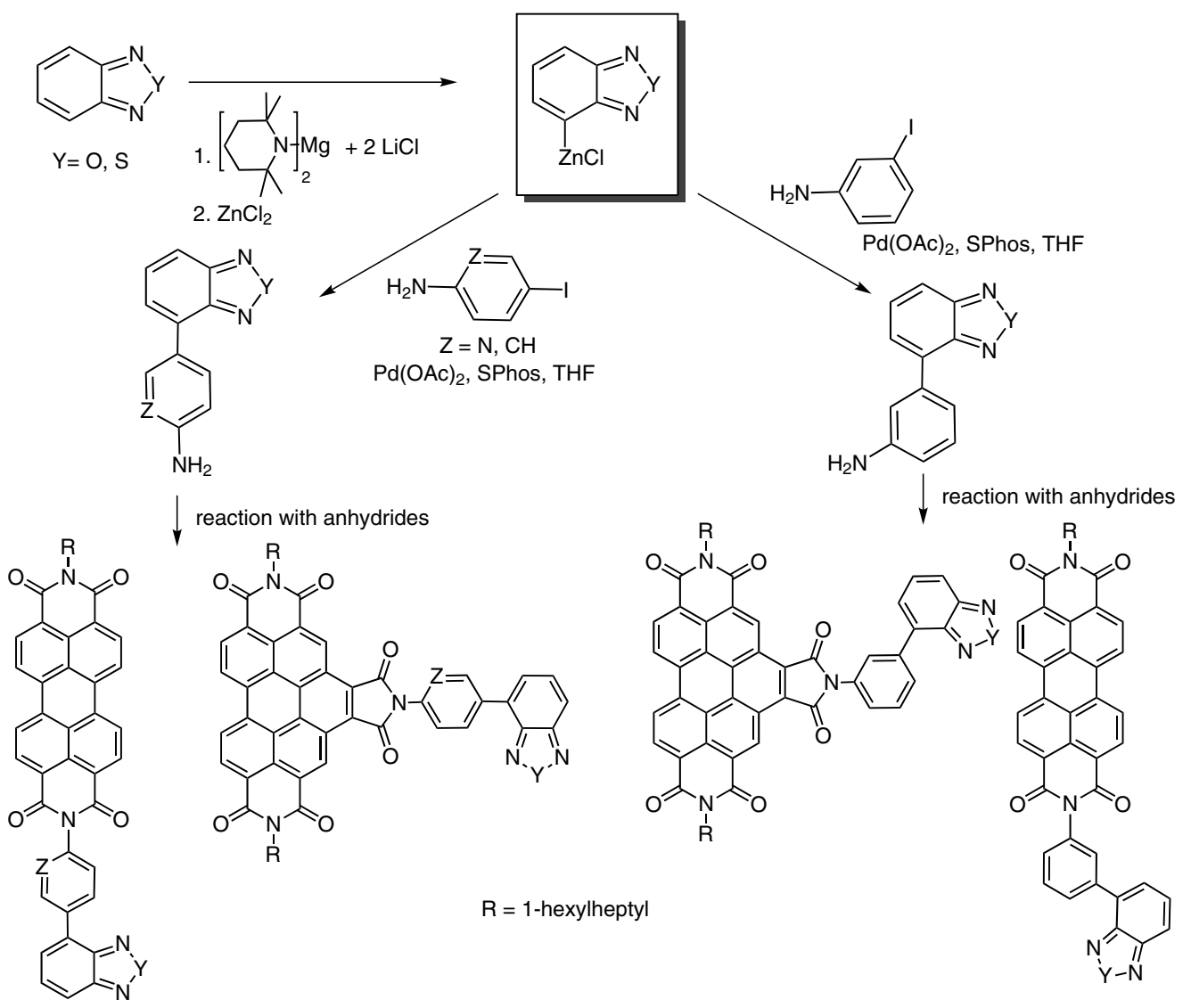

Significance: This work reports efficient synthetic procedures for the connection of two of the best electron-acceptor units (benzothiadiazoles or benzoxadiazoles and perylenes dimides) to create structures that are of irregular shape that do not crystallize. The combined cross-coupling and condensation yields allow for quantities of materials to be produced and the authors also demonstrated direct cross-coupling of the key zinc reagent on functional perylene dimides.
Comment: Organic semiconductors are often insoluble crystalline materials that are difficult to process. Crystalline materials are problematic for the formation of devices because mechanical or thermal stress can create cracks in the structures. As a result, general routes to molecular semiconductors with amorphous solid-state structures are desirable. It will be interesting to see, if the transport properties of these materials compare to crystalline materials. 